\title{
Eosinophilic Fasciitis With a Malignant Outcome
}

\author{
Salman Ansari ${ }^{\mathrm{a}}$, Umair Iftikhar ${ }^{\mathrm{b}}$, Asma Jamil ${ }^{\mathrm{c}}$, Aamir Ansaric ${ }^{\mathrm{c}}$, \\ Syed Iftikharc, d
}

\begin{abstract}
Eosinophilic fasciitis (EF) is an uncommon localized fibrosing disorder affecting the fascial layers of the human body. To date less than 300 cases of EF have been reported worldwide. Due to the limited prevalence, extensive studying of its pathogenesis and treatment has not yet been established. Furthermore, little is known regarding the long-term prognosis and comorbidities associated with EF. In this case study, we discuss a 72-year-old female patient who was diagnosed with EF and subsequently developed squamous cell carcinoma (SCC) of the skin in areas of previously exposed EF. This case represents the first reported case of SCC of the skin in a patient with previously active EF in the same area of skin.
\end{abstract}

Keywords: Eosinophilic fasciitis; Squamous cell carcinoma

\section{Introduction}

Eosinophilic fasciitis (EF), also known as Shulman syndrome, is one of the most unexplored diseases in medicine due to its rarity. When first discovered in 1974, EF was considered to be a scleroderma mimicker [1]. Both present with features of skin fibrosing and an elevated erythrocyte sedimentation rate (ESR). However, the presence of elevated eosinophils, sparing of hands and feet (hence no sclerodactyly and nailfold capillary changes), less internal organ involvement and the absence of Raynaud's phenomenon aids in differentiating these two diseases.

$\mathrm{EF}$ is likely an autoimmune process. The underlying pathophysiology is believed to be from increased expression of fibronectin and collagen [2]. Furthermore, elevated levels

Manuscript submitted January 29, 2021, accepted February 12, 2021

Published online March 24, 2021

aDepartment of Biochemistry, University of California, Santa Barbara, CA, USA

${ }^{b}$ Department of Education, Carden Arbor View School, Upland, CA, USA

'Department of Medicine, University of California, Riverside School of Medicine/Riverside Community Hospital, Riverside, CA, USA

${ }^{\mathrm{d} C o r r e s p o n d i n g ~ A u t h o r: ~ S y e d ~ I f t i k h a r, ~ D e p a r t m e n t ~ o f ~ M e d i c i n e, ~ U n i v e r s i t y ~}$ of California, Riverside School of Medicine/Riverside Community Hospital, Riverside, CA, USA. Email: Syed.iftikhar@hcahealthcare.com

doi: https://doi.org/10.14740/jmc3665 of circulating eosinophils support an inflammatory process. Elevated activity of interleukin-2, and interferon- $\gamma$ also points to an accentuated inflammatory response [2]. EF is characterized by the thickening of the dermis and subcutaneous fascia [3]. This skin induration can lead to the affected skin area having an orange peel-like or cobblestone-like appearance and "groove" sign caused by linear depression along the course of vascular structures [4]. Prayer sign due to tenosynovitis of hands is seen in $56 \%$ cases but is non-specific and can be found in other sclerodermiform disorders as well [4]. Due to this ongoing inflammation, some hypothesize that there could be an increased risk of certain cancers. Association with hemato-lymphoid malignancies has been reported in the literature [5]. However to date, no such association with skin cancers such as squamous cell carcinoma (SCC) has been identified. We discuss a patient who presented with EF and subsequently developed SCC of the skin. As more cases of EF are reported, we believe association with skin cancers such as SCC should be explored.

\section{Case Report}

A 72-year-old female was initially found to have morphea on the skin 4 months prior to presentation with 3 months of progressive thickening of the skin on the ankle, chest, back, arms, and neck. The findings were diffuse, and there was no fever, myalgias or arthralgias. The skin changes were asymmetric and not associated with any pain or pruritis; however they had been progressively worsening over the past 3 months (Fig. 1). She reported decreased mobility of her extremities as a result. There were no other identifiable pertinent systemic manifestations outside of the skin. This presentation was associated

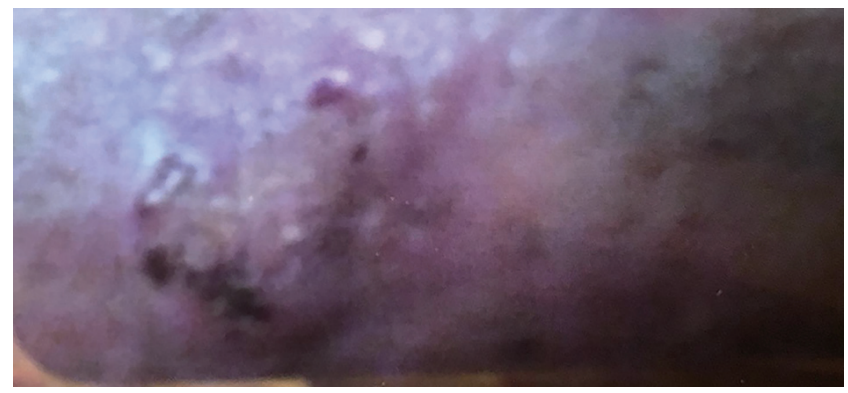

Figure 1. Posterior aspect of the patient's leg showing fibrosing and skin thickening. The biopsy revealed this to be consistent with eosinophilic fasciitis. 


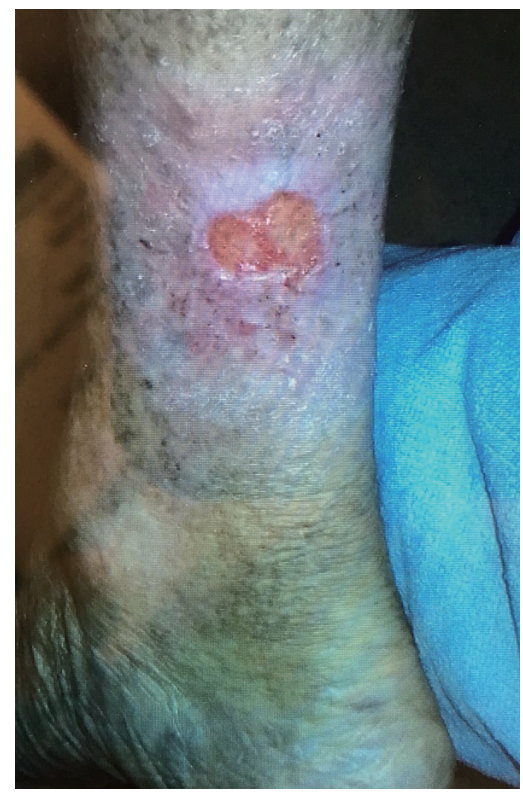

Figure 2. Ulceration of right posterior aspect of the right leg. The biopsy revealed this lesion to be squamous cell carcinoma.

with an elevated ESR, but negative antibodies for lupus, scleroderma and other autoimmune processes. As a result, a skin and muscle biopsy was done and the findings revealed a thickened fascial layer associated with a lymphocytic infiltrate. The patient was started on $60 \mathrm{mg}$ of prednisone and was prescribed the medication from April 2013 to March 2014. The patient had improvements in skin thickening throughout the body and was started on topical fluocinolone acetonide $0.01 \%$ once oral steroids were discontinued.

The patient was re-evaluated in the year of 2018. Her skin thickening showed improvement and had a return of nearly normal ankle motion. She was able to walk downstairs normally and exercise comfortably. She took precautions such as limited sun exposure and decreased strenuous exercise. However she noticed that some areas of skin thickening had returned after 3 months on the posterior aspect of the right lower extremity. She attempted use of topical corticosteroid; however the lesion did not resolve and within a few months, this lesion became ulcerated (Fig. 2). The patient underwent biopsy which revealed SCC. After that, wide and deep excision skin biopsy involving muscular layer was performed (Fig. 3). Wound care was able to reduce the size to a superficial ulcer of about $2 \times 3 \mathrm{~cm}$ diameter with good granulation tissue. She returned for routine follow-up without signs of recurrence of EF or carcinoma.

\section{Discussion}

EF generally occurs equally across both sexes in middle-aged adults. EF is considered to be idiopathic, but researchers believe that abnormal immune system response or inflammatory reaction may play a pivotal role in causing the disease. EF may be associated with tumor growth factors such as trans-

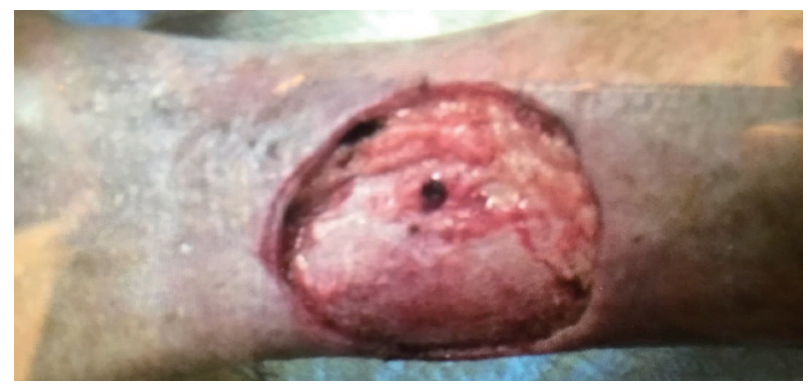

Figure 3. Wide excision following detection of squamous cell carcinoma of the skin.

forming growth factor-beta (TGF- $\beta$ ), interleukin-1, 2, 5, 10, interferon- $\gamma$ and leukemia inhibitory factor (LIF). High level of LIF prevents final differentiation stages of the cells [6]. TGF- $\beta$ activates fibroblasts resulting in myofacial plane thickening and subsequent fibrosis [7]. Cases that are preceded by trauma are thought to be a result of increased antigenicity of fascia and subcutaneous tissue [8].

Lab findings usually are significant for elevated ESR, hypergammaglobulinemia and peripheral eosinophilia. Of note, lab findings do not always correlate with the disease activity.

Hall mark of EF is the presence of subcutaneous tissue and fascia thickening on deep wedge tissue biopsy. Histologic findings change as the disease progresses from initial stage where inflammatory cells such as plasma cell, lymphocyte and eosinophil predominate to late stage when skin and fascia is thickened and collagenized. Magnetic resonance imaging (MRI) shows facial thickening of the subcutaneous tissue and can be used if the biopsy is inconclusive and for treatment monitoring.

$\mathrm{EF}$ association with hematologic malignancies has been described in literature. One study cited $10 \%$ of patients have associated myeloproliferative disorder and leukemia including chronic lymphocytic and myelomonocytic leukemia. EF has been found to be associated with other autoimmune conditions such as Hashimoto's thyroiditis, diabetes mellitus and autoimmune cytopenias. Previous studies have shown that exposure to certain medications (phenytoin), strenuous exercise, or trauma, post radiation, borrelia burgdorferi infection, post insect bite and asthma may be the triggering factors for EF.

Commonly reported onset symptoms of the disease include the inflammation of the fascia, with the arms and legs being the most prominent areas affected by the disease. At this early stage, patients with the disease may experience stiffness and pain after exercising. The disease then progresses to extreme cutaneous changes like peau's orange and woody induration of the skin. Currently, there is no agreed-upon therapy or treatment for EF. Systemic steroid therapy is usually initial treatment of choice and is effective in $70 \%$ cases [9-12]. Other treatment options included D-pencillamine, cyclosporine, azathioprine, chloroquine and infliximab. Given no controlled trials are available for treatment and spontaneous remission can occur, it is difficult to assess success of the treatment [13].

Very few cases have reported an association between EF and malignancies. Isolated cases have been reported includ- 
ing development of breast cancer in two sisters with EF [14]. The most common association with EF is that of hematologic malignancies such as leukemia. However, there have been no documented studies of EF being associated with skin cancer such as SCC. SCC of the skin is one of the most common skin malignancies and results from the uncontrolled growth of cells in the epidermis. The underlying hypothesized etiology of SCC is the p53 mutation. This is often caused by exposure to ultraviolet rays (UV) radiation. Other factors that can lead to SCC are human papilloma virus infection, severe burn scar, chronic ulcer and immunosuppressed state [15]. It is unknown if the underlying disease process itself or the subsequent pharmacologic immunosuppression which is used to treat certain diseases such as organ transplants is the underlying etiology for development of cutaneous cancers [16]. The same proposed mechanism can be applied to EF and the potential development of cutaneous cancers. It is possible that long-term steroid use in EF patients predisposes them to cutaneous malignancies. The second hypothesis that can be postulated is that EF can lead to various cancers by itself. More research needs to be conducted to evaluate this association.

\section{Conclusion}

$\mathrm{EF}$ is an autoimmune cutaneous disease that still remains unclear in both its etiology and pathogenesis. This case highlights a potential association between EF and SCC.

\section{Acknowledgments}

None to declare.

\section{Financial Disclosure}

None to declare.

\section{Conflict of Interest}

None to declare.

\section{Informed Consent}

Informed consent was privately obtained and documented.

\section{Author Contributions}

All authors have contributed to the manuscript. Salman Ansari: conceptualization, methodology; Umair Iftikhar: writing of original draft, review and editing; Asma Jamil and Aamir Ansari: visualization, review and editing. Syed Iftikhar: final draft editing and supervision. All authors read and approved the final version of the manuscript.

\section{Data Availability}

The authors declare that data supporting the findings of this study are available within the article.

\section{References}

1. Jarratt M, Bybee JD, Ramsdell W. Eosinophilic fasciitis: an early variant of scleroderma. J Am Acad Dermatol. 1979;1(3):221-226.

2. Jinnin M, Yamamoto T, Asano $\mathrm{Y}$, Ishikawa $\mathrm{O}$, Sato S, Takehara K, Hasegawa M, et al. Diagnostic criteria, severity classification and guidelines of eosinophilic fasciitis. J Dermatol. 2018;45(8):881-890.

3. Viallard JF, Taupin JL, Ranchin V, Leng B, Pellegrin JL, Moreau JF. Analysis of leukemia inhibitory factor, type 1 and type 2 cytokine production in patients with eosinophilic fasciitis. J Rheumatol. 2001;28(1):75-80.

4. Kirchgesner T, Dallaudiere B, Omoumi P, Malghem J, Vande Berg B, Lecouvet F, Houssiau F, et al. Eosinophilic fasciitis: typical abnormalities, variants and differential diagnosis of fasciae abnormalities using MR imaging. Diagn Interv Imaging. 2015;96(4):341-348.

5. Naschitz JE, Misselevich I, Rosner I, Yeshurun D, Weiner P, Amar M, Amato L, et al. Lymph-node-based malignant lymphoma and reactive lymphadenopathy in eosinophilic fasciitis. Am J Med Sci. 1999;318(5):343-349.

6. Helfman T, Falanga V. Eosinophilic fasciitis. Clin Dermatol. 1994;12(3):449-455.

7. Ihn H. Eosinophilic fasciitis: from pathophysiology to treatment. Allergol Int. 2019;68(4):437-439.

8. Morgan ND, Hummers LK. Scleroderma mimickers. Curr Treatm Opt Rheumatol. 2016;2(1):69-84.

9. Chun JH, Lee KH, Sung MS, Park CJ. Two cases of eosinophilic fasciitis. Ann Dermatol. 2011;23(1):81-84.

10. Urzal J, Cimbron M, Mendonca T, Farinha F. Eosinophilic fasciitis (Shulman's disease): review and comparative evaluation of seven patients. Reumatologia. 2019;57(2):85-90.

11. Bennett RM, Herron A, Keogh L. Eosinophilic fasciitis. Case report and review of the literature. Ann Rheum Dis. 1977;36(4):354-359.

12. Bischoff L, Derk CT. Eosinophilic fasciitis: demographics, disease pattern and response to treatment: report of 12 cases and review of the literature. Int J Dermatol. 2008;47(1):29-35.

13. Lebeaux D, Frances C, Barete S, Wechsler B, Dubourg $\mathrm{O}$, Renoux J, Maisonobe T, et al. Eosinophilic fasciitis (Shulman disease): new insights into the therapeutic management from a series of 34 patients. Rheumatology (Oxford). 2012;51(3):557-561.

14. Tsoi KL, Custers M, Bij de Vaate L, Jacobs JW. Eosinophilic fasciitis. BMJ Case Rep. 2012;2012.

15. Howell JY, Ramsey ML. Cancer, squamous cell of the skin. StatPearls Publishing. 2019.

16. Herman S, Rogers HD, Ratner D. Immunosuppression and squamous cell carcinoma: a focus on solid organ transplant recipients. Skinmed. 2007;6(5):234-238. 